\title{
Lung fibroblasts from patients with emphysema show a reduced proliferation rate in culture
}

\author{
O. Holz, I. Zühlke, E. Jaksztat, K.C. Müller, L. Welker, M. Nakashima, K.D. Diemel, D. Branscheid, \\ H. Magnussen, R.A. Jörres
}

Lung fibroblasts from patients with emphysema show a reduced proliferation rate in culture. O. Holz, I. Zühlke, E. Jaksztat, K.C. Müller, L. Welker, M. Nakashima, K.D. Diemel, D. Branscheid, H. Magnussen, R.A. Jörres. (C) ERS Journals Ltd 2004.

ABSTRACT: Emphysema is characterised by a loss of alveolar structure, as reflected in elastic recoil and gas exchange. As fibroblasts play a key role in the maintenance of structure, the current authors hypothesised that their proliferation might be constitutively impaired in lung emphysema.

Using explant cultures, lung fibroblasts were obtained from resected lungs of $\mathbf{1 0}$ patients with emphysema (median forced expiratory volume in one second (FEV1) 40\% predicted) and 10 control patients (FEV1, 95\% pred). The doubling time (DT) was measured over 4 days under standard conditions $(10 \%$ foetal calf serum) prior and after cryopreservation. Additionally, in seven samples per group the total population doubling level (PDL) was determined.

In emphysema, mean \pm SEM DT was $33.6 \pm 2.8 \mathrm{~h}$ compared with $24.8 \pm 1.4 \mathrm{~h}$ in controls. The differences in DT were preserved after cryopreservation. Groups also differed in the initial slope of the PDL plot during long-term culture (up to 35 days). However, the median (range) maximum PDL did not differ significantly between groups (13.8 (7.4-22.6) versus $20.2(11.2-25.5))$.

The current authors, therefore, suggest that the reduced proliferation rate in vitro of lung fibroblasts from patients with emphysema reflects a persistent, intrinsic failure of cellular replacement and maintenance in this disease, possibly in relation to pre-term aging.

Eur Respir J 2004; 24: 575-579.
Hospital Großhansdorf, Center for Pneumology and Thoracic Surgery, Großhansdorf, Germany.

Correspondence: O. Holz, Research Laboratory, Hospital Großhansdorf, Centre for Pneumology and Thoracic Surgery, Wöhrendamm 80, D-22927 Großhansdorf, Germany. Fax: 494102692295

E-mail: o.holz@pulmoresearch.de

Keywords: Cell culture

emphysema

fibroblast

human

lung structure

proliferation

Received: December 302003

Accepted after revision: June 232004

This research has been supported by Landesversicherungsanstalt (LVA), Freie und Hansestadt, Hamburg, Germany, and in part by a grant from AstraZeneca, Germany.
Destruction of alveolar walls and a resultant reduction of elastic recoil and surface area are hallmarks of lung emphysema and thought to be linked to neutrophilic inflammation and loss in protease/antiprotease and oxidant/antioxidant balance [1], as well as apoptosis of endothelial and epithelial cells [2]. Systemic factors such as a poor nutritional state [3] might also enhance emphysema as suggested by starving experiments in rodents $[4,5]$.

Initiation and progress of lung emphysema are closely linked to the inhalation of noxious agents such as cigarette smoke [1]. Except for the known genetic pre-disposition by $\alpha_{1}$ antitrypsin deficiency [1], it is unknown which factors explain the observation that only a minority of smokers develop emphysema [1]. Furthermore, there are diseases such as cystic fibrosis, in which extensive airway inflammation, neutrophilia and associated biochemical alterations are maintained over long periods of time, without provoking emphysema as a general outcome. In addition, from current knowledge the evidence of effects of anti-inflammatory interventions on the course of the disease in human subjects appears to be weak [6]. This suggests that, in addition to inflammation, other factors might be required in the pathogenesis of lung emphysema.

The integrity of the lung depends on extracellular matrix components, but also on fibroblasts that produce these compounds and provide structural support [7]. Structural changes in the lung shortly after cellular damage by apoptosis-inducing agents suggest a high rate of turnover of parenchymal cells to cope with the physical and chemical stresses occurring in the alveolar environment $[8,9]$. Therefore, homeostasis of alveolar septa and thus structural maintenance could be disrupted by a deterioration of cellular replacement. Based on this the current authors hypothesised that one of the differences between normal and emphysematous lungs might be an intrinsic loss in the proliferative activity of fibroblasts. This would provide a partial explanation for the apparently self-sustained progress of the disease, and potentially the clinical impression that many patients with emphysema appear to be aging ahead of time. If there should be an intrinsic impairment in proliferation, the difference against normal subjects should become apparent under standardised conditions that do not preserve the inflammatory environment existing in vivo [10, 11]. The current authors, therefore, isolated fibroblasts from parenchymal tissue of resected lung specimens of patients with and without emphysema and compared the rate and course of cell proliferation in culture.

\section{Material and methods}

\section{Patients}

A total of 10 patients with moderate to severe emphysema (Global Initiative for Chronic Obstructive Lung Disease 
(GOLD); stages IIa and IIb) [1], and 10 patients without clinical, morphological or functional signs of chronic obstructive pulmonary disease (COPD) or emphysema were included into the study (table 1). All but one patient underwent surgery for lung tumour resection and all patients were smokers except for two patients without COPD. There was no difference in tumour size or staging between groups. The diagnosis took into account the patient's history, symptoms, chest radiograph (emphysema: $\mathrm{n}=5$, control: $\mathrm{n}=7$ ), computed tomography (emphysema: $\mathrm{n}=8$, control $\mathrm{n}=5$ ), histological findings, expiratory flow-volume curves, as well as resistance loops and lung hyperinflation determined in a body plethysmograph. Diffusion capacity for carbon monoxide was available for a number of patients (emphysema: $n=5$, control $n=3$ ). The consistency of the available information in each patient, indicating either the presence or absence of COPD with significant emphysema, was reflected in the separation of functional data between groups (table 1). The study had been approved by the local Ethics Committee and all patients gave their written informed consent.

\section{Lung fibroblasts}

The macroscopical appearence of resected specimens was examined and pleura-free parenchymal samples were taken from the very peripheral, noncancerous areas of the resected lung. Patients showing massive inflammation or visible/ palpable lung metastases were excluded. Samples were washed in Hank's buffered saline (Invitrogen; Karlsruhe, Germany), minced with a scalpel $\left(1-2 \mathrm{~mm}^{2}\right)$ and transferred onto $25 \mathrm{~cm}^{2}$ culture dishes (Sarstedt; Nürnbrecht, Germany) for primary explant culture in a humidified chamber at $37^{\circ} \mathrm{C}$,

Table 1.-Subjects' characteristics

\begin{tabular}{lcc}
\hline & Control & Emphysema \\
\hline Patient n & 10 & 10 \\
Age yrs & $63.5(54-67)$ & $65.5(58-70)$ \\
Sex & & \\
$\quad$ Male & 6 & 10 \\
$\quad$ Female & 4 & 0 \\
Height cm & $175.5(159.0-177.0)$ & $175.5(168.0-182.0)$ \\
Weight Kg & $75.5(62.0-80.0)$ & $65.5(65.0-82.0)$ \\
VC \% pred & $102.4(87.6-110.5)$ & $74.6(72.0-90.0)^{*}$ \\
FEV1 \% pred & $94.8(83.9-103.3)$ & $39.6(34.2-46.0)^{* *}$ \\
FEV1/VC \% pred & $95.8(94.2-102.0)$ & $49.4(44.1-54.0)^{* *}$ \\
FEV1/FVC \% & $78.7(76.3-79.4)$ & $39.3(34.0-44.7)$ \\
ITGV \% pred & $103.8(94.4-110.0)$ & $175.5(165.8-193.9)^{* *}$ \\
RV L & $2.3(2.0-2.5)$ & $4.6(4.3-6.3)^{* *}$ \\
TLC L & $6.6(4.8-7.1)$ & $8.4(7.5-9.9)^{* *}$ \\
RV/TLC \% & $41.6(32.6-45.2)$ & $59.1(52.3-65.0)^{* *}$ \\
GOLD n & & 0 \\
0 & 0 & 0 \\
I & 0 & 2 \\
IIa & 0 & 8 \\
IIb & 0 & 0 \\
III & 0 & $47.5(40-75)^{*}$ \\
Smoking history ${ }^{\#}$ pack-yrs & $22.5(20-30)$ & $31.2(29.3-40.9)^{* *}$ \\
DT h & $25.0(21.7-25.4)$ & $36.3(34.1-47.9)^{*}$ \\
DT cryopreserved ${ }^{+}$h & $29.0(26.0-36.8)$ & \\
\hline
\end{tabular}

Data presented as mean (interquartile range) unless otherwise stated. VC: vital capacity; FEV1: forced expiratory volume in one second; ITGV: intrathoracic gas volume; RV: residual volume; TLC: total lung capacity; GOLD: Global Initiative for Chronic Obstructive Lung Disease; DT: doubling time. ${ }^{\#}$ : estimated pack-yrs from patient files; ${ }^{+}: \mathrm{n}=8$ proliferation experiments in emphysema and $n=9$ in controls due to insufficient cell numbers. *: $\mathrm{p}<0.05 ; * *: \mathrm{p}<0.01$ regarding the difference between groups.
5\% carbon dioxide. Cells were grown in Dulbecco's modified Eagle's medium (DMEM), containing 10\% foetal calf serum (FCS; one batch used throughout the study), $100 \mathrm{U} \cdot \mathrm{mL}^{-1}$ penicillin, $100 \mu \mathrm{g} \cdot \mathrm{mL}^{-1}$ streptomycin, $50 \mu \mathrm{g} \cdot \mathrm{mL}^{-1}$ gentamycin (all cell culture reagents and antibiotics were obtained from Invitrogen). Primary culture was terminated prior to fibroblasts reaching confluence. Cells were trypsinised, one aliquot resuspended in medium plus $10 \%$ dimethylsulphoxide, stored for $24 \mathrm{~h}$ in $-80^{\circ} \mathrm{C}$ and then transferred into liquid nitrogen. Another aliquot was transferred to a fresh culture vessel (seeding density 4,000 cells $\cdot \mathrm{cm}^{-2}$ ). Further samples were grown for a minimum of 2 weeks in standard DMEM without antibiotics. None of the cultures showed signs of bacterial or fungal contamination during this period.

\section{Proliferation assay}

Proliferation was measured in passage two (3-4 population doublings after primary culture). Fibroblasts were transferred to 24-well dishes $\left(4,000\right.$ cells $\left.\cdot \mathrm{cm}^{-2}\right)$ and cell numbers determined manually after 24, 48, 72 and $96 \mathrm{~h}$. At each time point the average from 3 wells was taken. Mean cell numbers were logarithmically transformed and the linear regression slope was calculated to derive the doubling time (DT). Proliferation was also determined in cryopreserved cells. Proliferation experiments were performed $\sim 1$ week after thawing, to avoid a major increase in the number of cumulative population doublings compared with the experiments with fresh cells.

\section{Immunocytochemistry}

Cells of passage two were seeded onto microscope slides, incubated overnight. Prior to immunocytochemical analysis the slides were fixed in ice-cold acetone, air-dried and preincubated with Protein Block (Dako; Hamburg, Germany). After washing, slides were incubated with the antibodies anti-vimentin (Dianova; Hamburg, Germany), anti-pan cytokeratin (Dako), anti-smooth muscle actin (Dianova) and anti-CD45 (Dianova). Staining was performed using the Envision as well as New Fuchsin Staining Kits (Dako) according to the manufacturer's instructions. After counterstaining, slides were mounted and a minimum of 400 cells were scored for each antibody.

\section{Determination of apoptosis and necrosis}

Cells of passage two were seeded onto coverslips and grown for 2 days before staining with Annexin-V-Fluos staining Kit (Roche; Mannheim, Germany) according to the manufacturer's instructions. The number of fluorescent cells per field of view was determined and the total number of cells in the same field was counted by light microscopy.

In a further sample of cells, viability was evaluated using trypan blue exclusion after trypsinisation. Additionally, supernatants were collected after culture over 3 days to determine the concentration of lactate dehydrogenase (LDH).

\section{Long-term cell culture}

Cells from seven subjects of each group were thawed and seeded at standard density into passage one. The number of attached fibroblasts was determined by counting the cells in the supernatant. Starting at passage two, cells were seeded at 
100,000 cells per $25 \mathrm{~cm}^{2}$, cultured for 1 week with regular changes of medium, harvested with trypsin, counted and transferred to the next passage. Population doubling times were determined and weekly passaging was continued until the harvested cell numbers dropped below the initially seeded number of 100,000 . The sum of all weekly doublings yielded the maximum population doubling level (PDL). The initial slope of the growth curve was derived from the first 35 days (passage six) of culture. Following the same procedure additional experiments were performed using 1\% instead of $10 \%$ FCS

\section{Data analysis}

Owing to the skewed distribution of cell numbers and median values, interquartile ranges (IQR) were chosen for description and the Mann-Whitney U-test was employed for the comparison of groups. The initial steepness of population doubling curves was compared between groups by repeated measures ANOVA, with post hoc comparisons according to Newman-Keuls. Statistical p-values $<0.05$ were considered significant.

\section{Results}

The two groups of patients differed significantly in lung function but not in age and weight (table 1). Fibroblasts could be grown from all resected lungs. Most cells in passage two after primary culture displayed the typical fibroblast morphology and were positive for vimentin. The percentages of CD45+ cells in cultures from patients with emphysema and control patients were not significantly different from each other, median (IQR) values being $0.13(0-0.75)$ and $0(0-0) \%$. The same was true for the percentage of cytokeratin-positive cells, the respective values being $1.1(0.4-3.8)$ and 0.6 $(0.4-1.2) \%$. Cells were negative for smooth muscle actin. There was also no significant difference in viability after passage one, with values of 95 (93-97) and 98 (97-98)\%.

\section{Doubling time and apoptosis/necrosis}

The DT of fibroblasts from patients with emphysema was significantly higher than in control patients $(\mathrm{p}<0.001$, table 1 , figs 1,2 ). In two patients with emphysema and one control patient, cell numbers were insufficient for further experiments after cryopreservation but the difference in DT remained statistically significant ( $\mathrm{p}=0.05$, table 1$)$. Correspondingly, DT prior to and post-storage was significantly correlated $(\mathrm{r}=0.66$, $\mathrm{p}=0.004)$. There was no significant correlation between donor age and DT, neither in the whole set of patients $(\mathrm{r}=0.11)$ nor within groups (emphysema, $\mathrm{r}=-0.15$; controls, $\mathrm{r}=0.04$ ). Furthermore, the time spent in primary culture before the start of proliferation experiments did not differ significantly between groups. The DT of fibroblasts from the two nonsmokers in the control group was slightly lower than the median value of this group. In both groups no significant correlations between functional variables (table 1) and DT were observed.

The median percentages of apoptotic cells as determined on day 3 of the proliferation assays were $0.5(0-0.6) \%$ in emphysema and $0.8(0.1,1.7) \%$ in control patients (NS), and the concentration of $\mathrm{LDH}$ in cell culture supernatants was 59 $(56-70)$ and $58(56-70) \mathrm{U} \cdot \mathrm{L}^{-1}(\mathrm{NS})$.

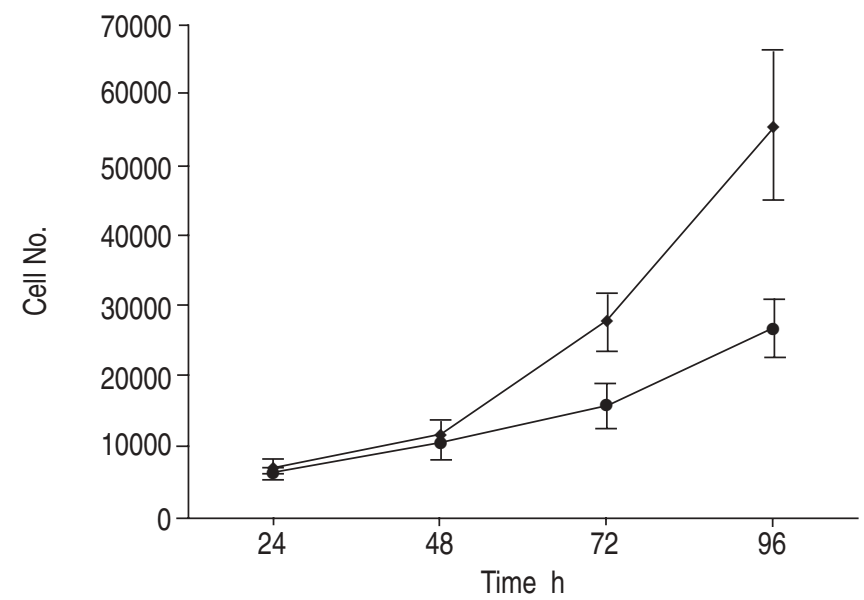

Fig. 1. - The mean \pm SEM cell numbers of fibroblasts from 10 patients with emphysema $(\diamond)$ and from 10 control patients ( $)$ during $96 \mathrm{~h}$ of culture. Data were derived from triplicate cultures in 24-well dishes. Fibroblast proliferation was significantly different between groups (analysis of variance, $\mathrm{p}=0.004$ ).

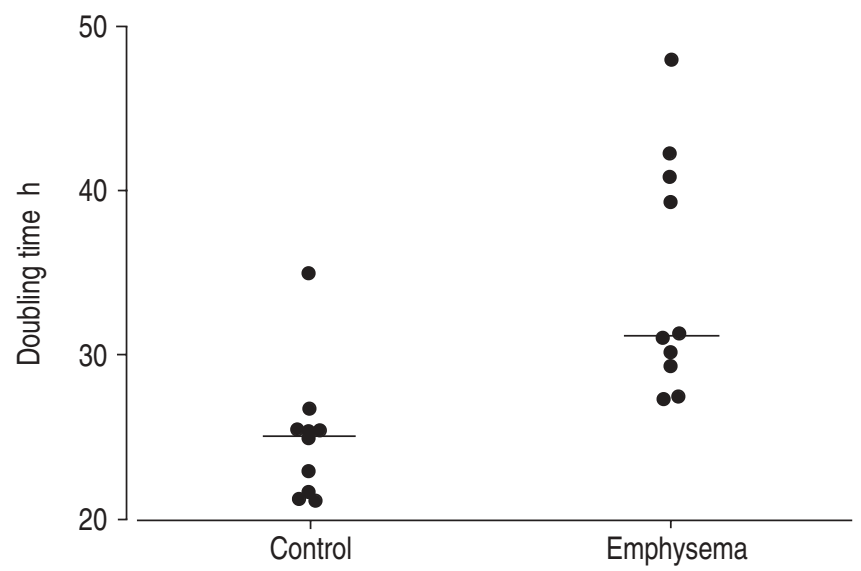

Fig. 2.-Doubling time (h) of lung fibroblast cultures which were studied in passage immediately after primary cultures and not previously stored in liquid nitrogen. Values were significantly different between groups (Mann-Whitney U-test, $\mathrm{p}<0.001$ )

\section{Population doubling level}

In seven patients from each group, cells were taken into long-term culture $(10 \%$ FCS $)$ and population doubling determined each week until proliferation ceased. Plots of PDL against time showed a steep curve in the early passages and a flattening with increasing time (fig. 3). The initial steepness was smaller in cultures from patients with emphysema compared with control patients $(\mathrm{p}<0.001$, ANOVA, fig. 3). Post hoc comparisons revealed that population doublings did not differ between groups on day $0(\mathrm{p}=0.94)$ and day $7(\mathrm{p}=0.17)$, but did on day $14,21,28$, and $35(\mathrm{p}=0.01$, $\mathrm{p}=0.0003, \mathrm{p}=0.0002, \mathrm{p}=0.0002$, respectively; fig. 3).

On day 42, four cultures from emphysema and five from control patients were still proliferating, on day 49 three and five, on day 56 three and four, and on day 63 two and one, cultures were proliferating respectively (fig. 3). The median (range) maximum PDL was 13.8 (7.4-22.6) days in fibroblasts from patients with emphysema and 20.2 (11.2-25.5) days in control patients $(\mathrm{p}=0.070$, one-tailed). In the experiments 

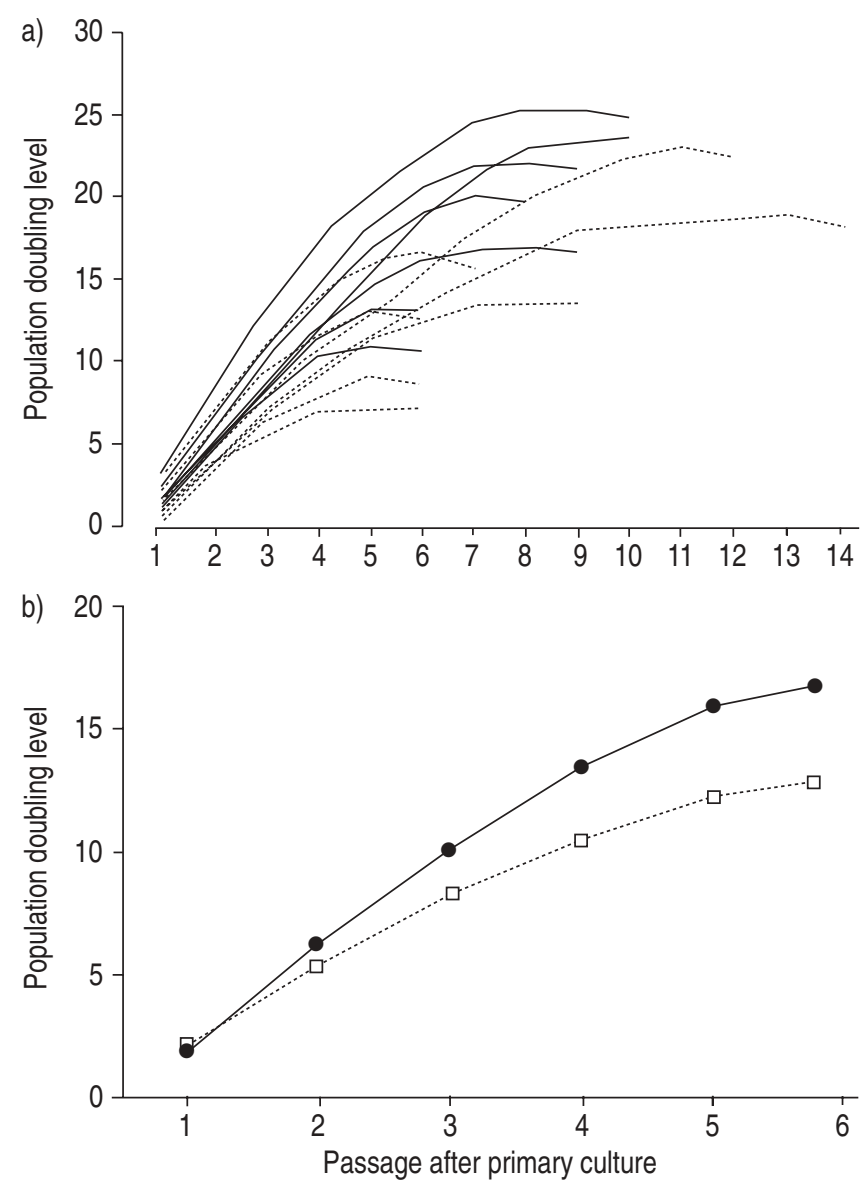

Fig. 3. - a) Population doubling level (PDL) plots of individual fibroblast cultures from seven control patients $(-)$ and seven patients with emphysema (- - - - ). Cell numbers were determined once a week and cells were grown until the number of cells harvested was lower than the number of cells seeded $(100,000)$. b) Results from the ANOVA analysis of the initial part of the PDL curves. To ensure comparability, data up to passage six (day 35), when all cultures were still replicating, were used for this analysis.

using culture medium with $1 \%$ FCS, the median (range) maximum PDL was $1.8(0.0-2.6)$ and 3.8 (0.8-13.1) days, respectively $(\mathrm{p}=0.032$, one-tailed $)$.

\section{Discussion}

In this study a reduced proliferation rate of cultured lung fibroblasts from patients with emphysema compared with control patients was observed. Correspondingly, the initial slope of the time course of population doublings in long-term cultures was lower. There was also a tendency towards a reduced number of total population doublings. The rates of apoptosis and necrosis were low and it seems unlikely that differences in these rates accounted for the differences in proliferation rate. The current findings suggest a change in fibroblast phenotype in emphysema. As this is maintained in vitro outside the inflammatory environment in vivo, it appears to be a persistent and constitutive feature of these cells, consistent with the hypothesis of a reduced capability of fibroblasts for cellular replacement in emphysema.

It has been recently suggested that one of the causes of the disrupted balance of alveolar septal homeostasis in emphysema could be an increased level of apoptosis of alveolar wall cells $[2,9,8]$. While these authors mainly focused on epithelial and endothelial cells [2], the current authors have chosen to study the lung fibroblast, based on the hypothesis that the fibroblast might be another key player in the development of emphysema. Fibroblasts are the major cell type found in the interstitium [12] and one of their primary roles is to maintain the integrity of the alveolar compartment by the turn-over of extracellular matrix components [7]. Two recent reports are in line with this hypothesis and the present results, suggesting an altered fibroblast phenotype in emphysema. In a sample of six patients overall, NoBUKUNI et al. [11] found a tendency towards reduced proliferation in emphysema under baseline conditions. Similarly, NoORDHOEK et al. [10] analysed fibroblasts from 16 patients, using bromo-deoxyuridine incorporation and DNA content on days 3 and 5 as outcome measures. Compared with the current authors' study, there were also differences in the severity of the disease, as well as in the culture medium, but again there was a trend towards reduced proliferation under baseline conditions, in line with the observations in the present study.

The difference in doubling time between groups was assessed in cells from passage two after primary culture (fig. 1, 2). It was also found in cells from the same patients after cryopreservation, which resulted in a small increase in doubling time and a spread of interquartiles, though the difference between groups was maintained. Within groups there was no association between fibroblast proliferation rate and functional parameters. This is not unexpected, since lung function represents an overall measure of impairment and it is very likely that with the progress of disease multiple factors have an influence on this. In the population doubling experiments (fig. 3), initial slopes also differed, lending further support to the assertion that proliferation rates were intrinsically different between emphysema and control. The current authors also found a trend towards a lower maximum PDL in fibroblasts from patients with emphysema, despite the large variability within groups. This was even statistically significant when using medium containing $1 \%$ FCS exerting less growth stimulation that $10 \% \mathrm{FCS}$. As the age distribution was very similar between groups, it is unlikely that this factor per se had a significant effect. In healthy subjects, a relationship between replicative life spans in culture and donor age does not seem to exist [13], although there is evidence that cells from donors with various diseases exhibit shortened life spans in culture compared with those from normal nondiseased donors [14].

It is tempting to hypothesise that the reduced proliferation rate and the tendency towards a lower maximum PDL in emphysema reflects a, possibly local, pre-term aging of cells. Conversely, it might be speculated that the prolonged need for repair due to cigarette smoke-induced damage has used up some of the limited capacity of fibroblasts to divide. The higher number of pack-yrs in patients with emphysema could be partially responsible for this. If aging of mesenchymal cells such as fibroblasts plays a role in the pathogenesis of emphysema, insight into this could help in the development of treatment strategies owing to the increasing knowledge in the field of stem cell research and tissue engineering. Although trials in human subjects are only at the beginning, some results in animal models of emphysema suggest that lung growth can be stimulated, e.g. by the use of retinoic acid derivatives [15, 16]. However, it is also possible that other epigenetic changes than aging are involved in or responsible for the reduced proliferation rate in emphysema. Conversley, caution is required in these arguments, as there are doubts whether replicative aging in vitro mimics the events that occur in vivo [17].

Similarly to the experiments of previous investigators $[10$, 11], the assays in the present study were performed under well-defined conditions after primary culture. It might be 
argued that the observed differences between groups resulted from differences occurring during the primary outgrowth of cells. Being aware of this potential bias, the current authors aimed to standardise the amount of tissue taken into culture, the final cell density and the duration of primary culture as much as possible. The time of primary culture did not differ between groups, despite the fact that the two cultures which showed the largest doubling times (both from emphysema patients), also spent the longest time in primary culture. It would be reasonable to expect that fibroblasts showing a slower proliferation in passage two also grew slower during primary culture. Unfortunately, it is not possible to monitor the division of individual cells without disturbing culture conditions. Thus, the possibility that in these two subjects lower numbers of cells grew out of tissue samples and that consequently cells had to undergo more divisions before entering the subsequent proliferation assay, cannot be ruled out. However, when the data of these two patients were excluded from the analysis, the difference in doubling time was still statistically significant $(\mathrm{p}=0.003)$.

To check that the differences in doubling times were based on differences in proliferation and not on differences in the rate of deceasing, cell viability and the percentages of necrotic and apoptotic cells during exponential growth and in confluent cultures were assessed. In all instances, low numbers of necrotic and apoptotic cells, high viability and no significant differences in the level of $\mathrm{LDH}$ in supernatants were observed, suggesting that potential differences in the rate of cell death between groups were small and unlikely to be cause the differences in the time course of cell numbers.

All experiments were performed in culture medium containing $10 \%$ FCS from a single batch, to ensure comparability. Commonly, $10 \%$ FCS is used for standard culture assays, but it is known that proliferation depends on the amount of serum [18]. The current authors chose $10 \%$ FCS as proliferation assays could be performed more accurately, owing to larger changes in cell numbers. However, the $10 \%$ FCS must be considered a strong nonspecific stimulation of fibroblasts and this has to be taken into consideration in the interpretation of results. In the long-term experiments population doublings were also assessed under 1\% FCS. While control subjects still showed a population doubling, although limited, there was hardly any proliferation in fibroblasts from patients with emphysema. This could reflect a difference between cells in the response to stimulation. In a previous study, fibroblasts from control subjects responded to a greater extent to stimulation with interleukin-1 $\beta$ by increasing proliferation and to transforming growth factor- $\beta 1$ by decreasing proliferation, compared with patients with emphysema [10].

In summary, the present data suggests that fibroblasts from patients with emphysema show a reduced proliferation rate in culture compared with cells from control subjects. As these differences were maintained under standardised conditions in vitro, outside the inflammatory environment encountered in vivo, they suggest a persistent alteration of lung fibroblasts. Any slow-down of the rate of proliferation, either unstimulated or stimulated, should impede cellular replacement and thereby aggravate an imbalance between tissue destruction and tissue renewal. In addition, the current data on population doubling levels provides evidence suggesting that factors reflecting pre-term aging are involved in this process. Further studies are needed to elucidate the molecular mechanisms underlying the, probably epigenetic, alteration of fibroblast phenotype in emphysema.

Acknowledgements. The authors would like to thank all patients for their co-operation and support during their research, in a situation in which quite understandably their major concern was different. In addition, the technical help of $\mathrm{K}$. Paasch and B. Feindt is gratefully acknowledged.

\section{References}

1. Fabbri LM, Hurd SS, for the GOLD Scientific Committee. Global strategy for the diagnosis, management, and prevention of COPD: 2003 update. Eur Respir $J$ 2003; 22: $1-2$.

2. Kasahara Y, Tuder RM, Cool CD, Lynch DA, Flores SC, Voelkel NF. Endothelial cell death and decreased expression of vascular endothelial growth factor and vascular endothelial growth factor receptor 2 in emphysema. Am J Respir Crit Care Med 2001; 163: 737-744.

3. Schols AM, Wouters EF. Nutritional abnormalities and supplementation in chronic obstructive pulmonary disease. Clin Chest Med 2000; 21: 753-762.

4. Sahebjami H, Wirman JA. Emphysema like changes in lungs of starved rats. Am Rev Respir Dis 1981; 124: 619-624.

5. Massaro D, Massaro GD, Baras A, Hoffman EP, Clerch LB. Calorie-related rapid onset of alveolar loss, regeneration, and changes in mouse lung gene expression. Am $J$ Physiol Lung Cell Mol Physiol 2003; 286: L896-L906.

6. Sherrill DL, Enright PL, Kaltenborn WT, Lebowitz MD. Predictors of longitudinal change in diffusing capacity over 8 years. Am J Respir Crit Care Med 1999; 160: 1883-1887.

7. Absher M. Fibroblasts. In: Massaro D. eds. Lung Cell Biology. Lung Biology in Health and Disease. New York, Marcel Dekker Inc, 1995; pp. 401-439.

8. Aoshiba K, Yokohori N, Nagai A. Alveolar wall apoptosis causes lung destruction and emphysematous changes. $\mathrm{Am}$ J Respir Cell Mol Biol 2003; 28: 555-562.

9. Tuder RM, Petrache I, Elias JA, Voelkel NF, Henson PM. Apoptosis and emphysema: the missing link. Am J Respir Cell Mol Biol 2003; 28: 551-554.

10. Noordhoek JA, Postma DS, Chong LL, et al. Different proliferative capacity of lung fibroblasts obtained from control subjects and patients with emphysema. Exp Lung Res 2003; 29: 291-302.

11. Nobukuni S, Watanabe $\mathrm{K}$, Inoue $\mathrm{J}$, Wen FQ, Tamaru N, Yoshida M. Cigarette smoke inhibits the growth of lung fibroblasts from patients with pulmonary emphysema. Respirology 2002; 7: 217-223.

12. Weibel ER, Gehr P, Haies D, Gil J, Bachofen M. The cell population of the normal lung. In: Bouhuys A. Lung cells in disease. Amsterdam, Elsevier and North Holland Biomedical Press, 1976; pp. 3-16.

13. Cristofalo VJ, Allen RG, Pignolo RJ, Martin BG, Beck JC. Relationship between donor age and the replicative lifespan of human cells in culture: a re-evaluation. Proc Natl Acad Sci USA 1998; 95: 10614-10619.

14. Oshima J, Campisi J, Tannock TC, Martin GM. Regulation of c-fos expression in senescing Werner syndrome fibroblasts differs from that observed in senescing fibroblasts from normal donors. J Cell Physiol 1995; 162: 277-283.

15. Massaro GD, Radaeva S, Clerch LB, Massaro D. Lung alveoli: endogenous programmed destruction and regeneration. Am J Physiol Lung Cell Mol Physiol 2002; 283: L305L309.

16. Rennard SI. Inflammation and repair processes in chronic obstructive pulmonary disease. Am J Respir Crit Care Med 1999; 160: S12-S16.

17. Wright WE, Shay JW. Historical claims and current interpretations of replicative aging. Nat Biotechnol 2002; 20: 682-688.

18. Jordana M, Schulman J, McSharry C, et al. Heterogeneous proliferative characteristics of human adult lung fibroblast lines and clonally derived fibroblasts from control and fibrotic tissue. Am Rev Respir Dis 1988; 137: 579-584. 